\title{
openheart Impact of invasive aortic pulse pressure on coronary microvascular endothelial- independent dysfunction and on mortality in non-obstructive coronary artery disease
}

\author{
Hyun Woong Park, ${ }^{1,2}$ Michel Corban, ${ }^{1}$ Takumi Toya, ${ }^{1,3}$ Ali Ahmad, ${ }^{1}$ Ilke Ozcan, \\ Lilach Lerman (D) , ${ }^{4}$ Amir Lerman (iD ${ }^{1}$
}

\begin{abstract}
- Additional supplemental material is published online only. To view, please visit the journal online (http://dx.doi.org/10. 1136/openhrt-2021-001925).

To cite: Park HW, Corban M, Toya T, et al. Impact of invasive aortic pulse pressure on coronary microvascular endothelial-independent dysfunction and on mortality in non-obstructive coronary artery disease. Open Heart 2022;9:e001925. doi:10.1136/ openhrt-2021-001925
\end{abstract}

Received 29 November 2021 Accepted 9 January 2022

D) Check for updates

(c) Author(s) (or their employer(s)) 2022. Re-use permitted under CC BY-NC. No commercial re-use. See rights and permissions. Published by BMJ.

'Department of Cardiovascular Medicine, Mayo Clinic Rochester, Minnesota, USA ${ }^{2}$ Department of Cardiovascular Disease, Chungnam National University Sejong Hospital, Sejong, Republic of Korea ${ }^{3}$ Division of Cardiology, National Defense Medical College, Tokorozawa, Saitama, Japan ${ }^{4}$ Division of Nephrology and Hypertension, Mayo Clinic, Rochester, Minnesota, USA

Correspondence to Dr Amir Lerman; Ierman.amir@ mayo.edu

\section{ABSTRACT}

Background Pulse pressure (PP), a raw index of arterial stiffness, is inversely related to coronary microvascular function, even among patients with non-obstructive coronary artery disease (CAD), as per non-invasive studies. We aimed to determine whether invasive aortic $\mathrm{PP}$ is associated with coronary microvascular endothelial dysfunction (CMED) and/ or coronary microvascular endothelial independent dysfunction (CMEID) in patients with non-obstructed CAD.

Methods We retrospectively analysed a cohort of 1894 patients (mean age, 51.2 years; 1261 (66.6\%) women) who presented with chest pain and non-obstructive CAD (angiographic stenosis $<50 \%$ ); coronary vasoreactivity was assessed in the mid-left anterior descending artery. The patients were classified based on whether aortic PP was high $(\geq 50 \mathrm{~mm} \mathrm{Hg}$ ). CMEID was defined as abnormal coronary flow reserve $(<2.5)$ or hyperaemic myocardial resistance $(>2.0$ $\mathrm{mm} \mathrm{Hg} / \mathrm{cm} / \mathrm{s}$ ), CMED was defined as abnormal acetylcholineinduced per cent change of coronary blood flow $(\leq 50 \%)$. Results Patients with high aortic PP had a higher rate of CMEID than those with low aortic PP (40.9 vs $25.2 \%$, $\mathrm{p}<0.001)$. Conversely, aortic PP was not associated with CMED. On multivariate analysis, high aortic PP was associated with CMEID occurrence (0R 1.42, 95\% Cl 1.13 to $1.78 ; \mathrm{p}=0.003$ ). On follow-up (median, 150 months), all-cause death was more frequent among patients with vs without high aortic $\mathrm{PP}(20.1 \%$ vs $7.3 \%$, log-rank $p<0.001)$ and $\mathrm{HR}$ was $2.08(95 \% \mathrm{Cl} 1.34$ to $3.32, p=0.002$ ) on multivariate cox regression analysis. Furthermore, among patients with low aortic PP, CMEID was an independent risk factor for all-cause mortality (HR 2.04, 95\% Cl 1.01 to $4.16, p=0.048$ ).

Conclusion In patients with non-obstructive $C A D$, invasive aortic PP was significantly associated with CMEID, but not with CMED. High aortic PP was an independent predictor of all-cause mortality, but CMEID was an independent risk factor in patients with low aortic PP.

\section{INTRODUCTION}

The incidence of non-obstructive coronary artery disease (CAD) on coronary angiography is $20 \%-30 \%$ among patients who present with chest pain. ${ }^{1}$ Coronary

\section{Key questions}

What is already known about this subject?

- Brachial pulse pressure (PP) was associated with coronary microvascular dysfunction in nonobstructive coronary artery disease (CAD) and PP and coronary microvascular dysfunction were important surrogate marker or cardiovascular outcomes in patients with CAD.

- Invasively measured aortic PP was more strongly positively correlated with arterial stiffness.

What does this study add?

- Invasively measured aortic PP was associated with coronary microvascular endothelial independent dysfunction (CMEID) in non-obstructive CAD.

- The aortic PP was an independent predictor of allcause deaths, but CMEID increased the risk of allclause death only in patients with low aortic PP.

How might this impact on clinical practice?

- Elevated aortic PP can be one of the key roles in CMEID, and choosing medical treatment based on measurements of these risk factors can improve clinical outcomes in non-obstructive CAD patients deserves further study.

microvascular dysfunction (CMD) plays an important role in the presentation of cardiac symptoms and increased future events in patients with non-obstructive CAD. ${ }^{2}$ Elevated pulse pressure $(\mathrm{PP})$ is usually regarded as a manifestation of reduced arterial elastic properties and increased arterial stiffness. ${ }^{3}$ The Strong Heart Study reported that PP was more strongly associated with vascular hypertrophy and atherosclerosis than systolic blood pressure (SBP). ${ }^{4}$ Increased PP also leads to fatigue and increased fracture in the elastic components of the vessel wall and is more likely to induce intimal damage, resulting in atherosclerosis. ${ }^{5}$ Increased PP with elevated 
SBP is also associated with stress on cardiac work and increases myocardial oxygen demand, while low diastolic blood pressure (DBP) can be a limiting factor for coronary perfusion and ischaemia. ${ }^{6}$

Although previous studies have reported that PP was inversely related to CMD in patients with non-obstructive CAD, these studies used transthoracic echocardiography to measure coronary flow and velocity and measured non-invasive BP. Furthermore, the number of study participants was relatively small, and coronary endothelial function was not examined separately for coronary microvascular endothelial dysfunction (CMED) and coronary microvascular endothelium-independent dysfunction (CMEID). ${ }^{7-9}$

Therefore, this study aimed to evaluate the relationship between invasively measured arterial stiffness indices measured directly from invasive aortic PP and coronary vasoreactivity, including CMED and CMEID, in patients with non-obstructive CAD.

\section{METHODS}

\section{Study population}

A total of 1998 consecutive patients referred to the Mayo Clinic between January 1992 and November 2019 for cardiac catheterisation without evidence of significant obstructive CAD on initial angiography were enrolled. Patients were referred to the cardiology department by their physician for clinically indicated coronary angiography for evaluation of chest pain or abnormal stress test results. The decision to refer patients for the assessment of chest pain was based on the discretion of the attending physician. All patients had recurrent chest pain suspected to be of cardiac origin. A coronary vaso-reactive test was also performed on discretion of the attending physician following coronary angiography. Angiograms were reviewed prior to administration of any pharmacological agents. Non-obstructive CAD was defined as $<50 \%$ luminal diameter obstruction. ${ }^{10}$ The exclusion criteria were as follows: (1) patients who had significant angiographic stenosis of any coronary artery requiring revascularisation; (2) pregnant patients; (3) patients with advanced chronic kidney disease (estimated glomerular filtration rate, eGFR $\left.<30 \mathrm{~mL} / \mathrm{min} / 1.73 \mathrm{~m}^{2}\right)$; (4) patients with valvular heart disease; (5) patients without invasive aortic $\mathrm{BP}$ and (6) patients who underwent percutaneous coronary intervention (PCI) at the left anterior descending artery (LAD) due to acute myocardial infarction. Of all patients enrolled, 104 patients were excluded, so that a total of 1894 patients were consequently analysed. Clinical history, laboratory data and current medications were collected from a detailed chart review by investigators (AA and FS) who were blinded to the results of coronary vasomotor function testing. All-cause death data were ascertained by a combination of public and institutional databases, death certificates, mail surveys and telephone calls.

\section{Invasive aortic BP and coronary reactivity test}

Aortic BP was measured at the ascending thoracic aorta immediately prior to coronary angiography. The physician placed a catheter flushed with normal saline, and then performed a zero-balancing procedure. Aortic BP was then measured through the catheter automatically, and aortic PP was obtained by subtracting the aortic DBP from the aortic SBP. The Strong Heart Study reported that a high central PP $(\geq 50 \mathrm{~mm} \mathrm{Hg})$ was an independent predictor of adverse cardiovascular events ${ }^{11}$ Accordingly, the current study defined high aortic PP as PP $\geq 50 \mathrm{~mm}$ $\mathrm{Hg}$.

The procedure protocols for invasive coronary reactivity tests have been described in previous studies. ${ }^{12}$ Nitrate and calcium channel blockers were discontinued prior to testing. TheLAD was used as the target vessel for observation in this study. A 0.014-inch Doppler guide wire (Flowire, Volcano/Philips Corporation, Rancho Cordova, California, USA) was positioned in the middle portion of the left anterior descending artery. Acetylcholine (ACh)-induced coronary blood flow ( $\mathrm{CBF}$ ) changes and vasodilation were used to measure CMED. Therefore, CMED can be evaluated by the percentage change in $\mathrm{CBF}$ in response to Ach (\% $\triangle \mathrm{CBF}$ Ach). CMED was defined as a change in $\mathrm{CBF}$ of $\leq 50 \%$ in response to Ach. CMEID was defined as abnormal coronary flow reserve (CFR) $(<2.5)$ or hyperaemic microvascular resistance (HMR) $(>2.0 \mathrm{~mm} \mathrm{Hg} / \mathrm{cm} / \mathrm{s})$ induced by adenosine. ${ }^{13} 14$

HMR and baseline microvascular resistance (BMR) were used to assess microvascular resistance. HMR was defined as mean distal coronary artery pressure divided by average distal peak flow velocity (APV) at maximal hyperaemia, and BMR was defined as MAP divided by APV at baseline. CMD was defined as having either a CMED or CMEID. ${ }^{15}{ }^{16}$ CMD was defined as having CMED, CMEID or both. Epicardial endothelial dysfunction was defined as a percentage decrease in coronary artery diameter (\% $\triangle \mathrm{CAD}$ Ach $) \geq 20 \%$ in response to a maximum ACh dose. The percentage change in coronary artery diameter in response to nitroglycerine ( $\% \Delta$ CAD NTG) was measured between the baseline coronary artery diameter and the last coronary artery diameter after nitroglycerin administration.

\section{Patient and public involvement}

Participants contributed to the study but were not involved in determining the research question or outcome measures, nor were they involved in recruitment, design or implementation of the study.

\section{Statistical analysis}

Continuous variables are presented as mean $\pm \mathrm{SD}$, while categorical variables are reported as frequencies and percentages. Normal distribution of measurements was confirmed using the Kolmogorov-Smirnov test. The Student's unpaired t-test for parametric continuous variables and the Wilcoxon signed-rank test for nonparametric continuous variables were used. Comparisons 
between categorical variables were performed using the Pearson $\chi^{2}$ test or Fisher's exact test, as appropriate.

Risk factors [old age ( $\geq 60$ years old), female sex, high body mass index (BMI) $\left(\geq 30 \mathrm{~kg} / \mathrm{m}^{2}\right)$, hypertension (HTN), diabetes mellitus (DM), dyslipidaemia, renal dysfunction $\left(<60 \mathrm{~mL} / \mathrm{min} / 1.73 \mathrm{~m}^{2}\right)$ and current smoking], and high aortic PP were evaluated using univariate and multivariate logistic regression analyses for predicting the incidence of CMEID and CMED, respectively. We also analysed the association of aortic $\mathrm{PP}$, measured as a continuous variable, with CMEID using the logistic regression model. Logistic regression analysis provided ORs and 95\% CIs. We compared two or more ROC curves using the area under the curve (AUC) comparison analysis method, as described by DeLong et $a l$. We also calculated integrated discrimination improvement (IDI) and net reclassification improvement (NRI) with a category-free option.

Multivariate Cox proportional hazard analysis was performed to identify independent correlates of all-cause death and to adjust for known potential confounders (high aortic PP, CMEID, old age, female sex, high BMI, HTN, DM, dyslipidaemia, renal dysfunction and current smoking status).

According to the profiles of aortic PP, cumulative allcause death was constructed using Kaplan-Meier estimates and compared using the log-rank test. A $p<0.05$ was considered statistically significant, and statistical analyses were performed using R V.4.0.3 (The R Foundation for Statistical Computing, Vienna, Austria).

\section{RESULTS}

\section{Baseline characteristics}

The study population included 1894 patients with nonobstructive CAD who underwent invasive physiology tests.

The clinical characteristics of the study groups are presented in table 1 . In general, the patients with high aortic PP $(n=1803)$ showed higher risk profiles than those with low aortic PP $(n=872)$ and were more likely to be women, have higher high-density lipoprotein and had a tendency for lower eGFR. Patients with high aortic PP had a higher atherosclerotic cardiovascular disease (ASCVD) risk score $(2.3$ vs $7.5 \%, \mathrm{p}<0.001)$ than patients with low aortic PP.

\section{Determinants of CMEID}

The high aortic PP group had lower CFR, higher HMR and higher base APV. Abnormal CFR $(<2.5)$ and abnormal HMR $(>2.0 \mathrm{~mm} \mathrm{Hg} / \mathrm{cm} / \mathrm{s})$ were also more common in the high aortic PP group. Patients with high aortic PP had a greater prevalence of CMEID than those with low aortic PP (40.9\% vs $25.2 \%$, OR $2.05,95 \%$ CI 1.69 to $2.50, \mathrm{p}<0.001$ ) (online supplemental table).

Multivariate analysis identified high aortic PP (OR 1.42, 95\% CI 1.13 to $1.78, \mathrm{p}=0.003$ ), old age (OR $1.79,95 \%$ CI 1.40 to $2.33, \mathrm{p}<0.001$ ), female sex (OR $1.29,95 \%$ CI 1.03 to $1.61, \mathrm{p}=0.027$ ), renal dysfunction (OR $1.41,95 \%$
CI 1.05 to $1.88, \mathrm{p}=0.021$ ) and current smoking (OR 0.77, $95 \%$ CI 1.40 to $2.30, \mathrm{p}<0.001)$ as the major determinants of CMEID occurrence (table 2).

Figure 1 shows the OR (95\% CI) for risk of CMEID, by aortic PP, as a continuous variable, after adjustment of the covariates at baseline: old age, female sex, high BMI, HTN, DM, dyslipidaemia, renal dysfunction and current smoking status. An increase in aortic PP was significantly non-linear associated with risk in CMEID (per $1 \mathrm{~mm} \mathrm{Hg}$ increase, OR: 1.02 (95\% CI 1.01 to 1.02 ), $\mathrm{p}<0.001$ ).

We conducted an ROC curve analysis of the predictive models for CMEID with risk factors (defined old age renal dysfunction, sex), aortic PP and risk factors plus aortic PP. Based on the results of differences among the AUCs, conventional risk factors in addition to aortic PP were significantly more predictive of CMEID than conventional risk factors alone (figure 2). Additionally, to estimate the incremental value of aortic PP in predicting CMEID, we compared the probabilities of events and nonevents of models using the relative IDI and category-free NRI (table 3). The IDI of adding aortic to the risk factors improved significantly (relative IDI $=1.23, \mathrm{p}<0.001$ ). The addition of aortic PP to the risk factors resulted in a significant improvement in the category-free NRI (total NRI 0.0499, $\mathrm{p}<0.011)$.

\section{Determinants of CMED}

There was no difference in $\% \Delta \mathrm{CBF}$ Ach and $\% \triangle \mathrm{CAD}$ Ach between the aortic PP groups. The incidence of CMD was not different between the two groups (online supplemental table).

On univariate analysis, high aortic PP was not associated with the risk of CMED $(51.1 \%$ vs $53.9 \%$, OR 1.12 , 95\% CI 0.93 to $1.34, \mathrm{p}=0.224)$. On multivariate analysis, high aortic PP was not an independent predictor of CMED. Old age (OR 1.43, 95\% CI 1.12 to $1.83, \mathrm{p}=0.005$ ), high BMI (OR 1.40, 95\% CI 1.16 to $1.71, \mathrm{p}=0.001$ ) and current smoking status (OR 0.64, 95\% CI 0.48 to $0.85, \mathrm{p}=0.002$ ) were independent determinants of CMED occurrence (table 4).

\section{All-cause deaths}

The median follow-up duration was 150 months (IQR 81-210 months). Clinical endpoints constituting all-cause mortality occurred in 136 patients (7.2\%).

Those with 'high aortic PP' had significantly higher cumulated rate of all-cause death than did those with low aortic PP (20.1\% vs $7.3 \%$; HR 2.51, 95\% CI 1.69 to 3.71, $\mathrm{p}<0.001, \log$-rank $\mathrm{p}<0.001$ ) (figure 3A). CMEID also increased the cumulative rate of death compared with those without CMEID (18.5\% vs $12.5 \%$; HR $1.56,95 \%$ CI 1.11 to $2.19, \mathrm{p}=0.010, \log$-rank $\mathrm{p}=0.010$ ) (figure $3 \mathrm{~B}$ ).

Following multivariate analysis, several risk factors including 'high aortic PP' (HR 2.08, 95\% CI 1.34 to 3.32, $\mathrm{p}=0.002$ ) were identified as the predictors of all-cause mortality in patients with non-obstructive CAD (table 4). Female sex (HR 0.64, 95\% CI 0.32 to $0.66, \mathrm{p}<0.001$ ) was a protective factor, and old age (HR 2.79, 95\% CI 
Table 1 Baseline characteristics according to aortic PP

\begin{tabular}{|c|c|c|c|c|}
\hline & All & Low aortic PP & High aortic PP & \\
\hline & $(n=1894)$ & $(n=856)$ & $(n=1038)$ & $P$ value \\
\hline Age, years & $51.2 \pm 12.4$ & $44.1 \pm 10.7$ & $57.1 \pm 10.6$ & $<0.001$ \\
\hline Female, n (\%) & $1261(66.6)$ & $445(52.0)$ & $816(78.6)$ & $<0.001$ \\
\hline $\mathrm{SBP}, \mathrm{mm} \mathrm{Hg}$ & $130.4 \pm 22.8$ & $113.2 \pm 11.7$ & $144.6 \pm 19.8$ & $<0.001$ \\
\hline $\mathrm{DBP}, \mathrm{mm} \mathrm{Hg}$ & $74.6 \pm 10.5$ & $72.7 \pm 9.4$ & $76.2 \pm 11.0$ & $<0.001$ \\
\hline Pulse pressure, $\mathrm{mm} \mathrm{Hg}$ & $55.8 \pm 18.4$ & $40.5 \pm 6.6$ & $68.4 \pm 15.2$ & $<0.001$ \\
\hline Hypertension, $\mathrm{n}(\%)$ & 801 (42.3) & $254(29.7)$ & $547(52.7)$ & $<0.001$ \\
\hline Diabetes mellitus, $n(\%)$ & $204(10.8)$ & $68(7.9)$ & $136(13.1)$ & $<0.001$ \\
\hline Dyslipidaemia, $n$ (\%) & $1046(55.2)$ & $408(47.7)$ & $638(61.5)$ & $<0.001$ \\
\hline Chronic kidney disease, $\mathrm{n}(\%)$ & $258(13.6)$ & $81(8.7)$ & $181(18.0)$ & $<0.001$ \\
\hline Previous Ml, n (\%) & $258(14.0)$ & $114(13.7)$ & $144(14.3)$ & 0.751 \\
\hline Previous vascular disease, $\mathrm{n}(\%)$ & $170(9.0)$ & $66(7.7)$ & $104(10.0)$ & 0.095 \\
\hline Current smoker, n (\%) & $228(12.0)$ & $138(16.1)$ & $90(8.7)$ & $<0.001$ \\
\hline Body mass index, $\mathrm{kg} / \mathrm{m}^{2}$ & $29.0 \pm 6.3$ & $29.1 \pm 6.3$ & $28.9 \pm 6.2$ & 0.469 \\
\hline Ejection fraction (\%) & $61.6 \pm 7.3$ & $61.3 \pm 7.1$ & $61.8 \pm 7.6$ & 0.226 \\
\hline $\mathrm{WBC}, 10^{3} / \mathrm{mm}^{3}$ & $6.8 \pm 2.1$ & $6.9 \pm 2.2$ & $6.6 \pm 2.1$ & 0.009 \\
\hline Haemoglobin, g/L & $135.5 \pm 14.2$ & $138.7 \pm 14.4$ & $132.8 \pm 12.3$ & $<0.001$ \\
\hline Total cholesterol, mg/dL & $186.2 \pm 43.1$ & $182.7 \pm 41.8$ & $189.0 \pm 43.9$ & 0.002 \\
\hline Trlglyceride, $\mathrm{mg} / \mathrm{dL}$ & $133.0 \pm 88.6$ & $131.7 \pm 89.4$ & $134.0 \pm 88.0$ & 0.591 \\
\hline $\mathrm{HDL}, \mathrm{mg} / \mathrm{dL}$ & $54.3 \pm 17.6$ & $51.2 \pm 17.0$ & $56.8 \pm 17.7$ & $<0.001$ \\
\hline $\mathrm{LDL}-\mathrm{C}, \mathrm{mg} / \mathrm{dL}$ & $105.5 \pm 37.2$ & $105.5 \pm 36.4$ & $105.5 \pm 37.9$ & 0.998 \\
\hline BUN, mg/dL & $14.8 \pm 4.9$ & $14.3 \pm 4.7$ & $15.2 \pm 5.0$ & 0.001 \\
\hline Creatinine, $\mathrm{mg} / \mathrm{dL}$ & $1.0 \pm 0.3$ & $1.0 \pm 0.3$ & $1.0 \pm 0.3$ & 0.044 \\
\hline eGFR (EPI), $\mathrm{mL} / \mathrm{min} / 1.73 \mathrm{~m}^{2}$ & $78.6 \pm 17.7$ & $84.1 \pm 16.8$ & $74.2 \pm 17.1$ & $<0.001$ \\
\hline NT-pro-BNP, pg/mL & $147.2 \pm 373.2$ & $122.5 \pm 326.2$ & $170.5 \pm 411.8$ & 0.096 \\
\hline hs-CRP, mg/L & $2.6 \pm 6.2$ & $2.9 \pm 6.5$ & $2.4 \pm 5.9$ & 0.205 \\
\hline Aspirin, $\mathrm{n}(\%)$ & $918(48.5)$ & $382(44.6)$ & $536(51.6)$ & 0.003 \\
\hline ACE or ARB, $n(\%)$ & $271(14.3)$ & $106(12.4)$ & $165(15.9)$ & 0.035 \\
\hline$\beta$-blocker, n (\%) & $579(30.6)$ & $229(26.8)$ & $350(33.7)$ & 0.001 \\
\hline Calcium channel blocker, n (\%) & $576(30.4)$ & $246(28.7)$ & $330(31.8)$ & 0.165 \\
\hline Nitrate, n (\%) & 750 (39.6) & $323(37.7)$ & $427(41.1)$ & 0.144 \\
\hline ASCVD risk score & $4.9 \pm 7.4$ & $2.3 \pm 3.3$ & $7.5 \pm 9.2$ & $<0.001$ \\
\hline
\end{tabular}

Continuous variables were expressed in mean $\pm \mathrm{SD}$ as indicated.

ARB, angiotensin II receptor blocker; ASCVD, atherosclerotic cardiovascular disease; BUN, blood urea nitrogen; DBP, diastolic blood pressure; eGFR, estimated glomerular filtration rate; EPI, epidemiology collaboration; HDL, high-density lipoprotein; hs-CRP, high sensitivity $\mathrm{C}$ reactive protein; LDL, low density lipoprotein; MI, myocardial infarction; NT-pro-BNP, N-terminal pro b-type natriuretic peptide; PP, pulse pressure; SBP, systolic blood pressure; WCC, white cell count.

1.85 to $4.19, \mathrm{p}<0.001)$, DM $(2.10,95 \%$ CI 1.31 to 3.37 , $\mathrm{p}=0.002$ ), renal dysfunction (HR $1.87,95 \%$ CI 1.25 to 2.79, $\mathrm{p}=0.002$ ), and current smoking (HR 2.46, 95\% CI 1.58 to $3.83, \mathrm{p}<0.001$ ) were independent predictors for all-cause mortality.

Subsequently, we evaluated the impact of CMEID on all-cause mortality according to the presence of 'high aortic PP'. Those with 'high aortic PP and CMEID' had significantly higher rate of all-cause deaths followed by 'high aortic PP without CMEID', 'low aortic PP with
CMEID' and 'low aortic PP without CMEID' (19.5\% vs $20.1 \%, 17.3 \%$, and $8.5 \%$, HR 2.22, 2.17, 1.89 , and reference 'Low aortic PP without CMEID') (figure 3C).

Among non-obstructive CAD patients with 'high aortic PP', CMEID was not associated with all-cause death $(19.5 \%$ vs $20.9 \%, \log$-rank $\mathrm{p}=0.562)$. Among 'low aortic PP' patients, subjects with CMEID showed significant, independently associated risk of all-cause death (HR 2.04, $95 \%$ CI 1.01 to $4.16, \mathrm{p}=0.049$ ) on multivariate analysis. 
Coronary artery disease

Table 2 Determinants of coronary microvascular endothelial independent dysfunction

\begin{tabular}{|c|c|c|c|c|c|c|}
\hline \multirow[b]{2}{*}{ Variables } & \multicolumn{3}{|c|}{ Univariate analysis } & \multicolumn{3}{|c|}{ Multivariate analysis } \\
\hline & OR & $95 \% \mathrm{Cl}$ & $P$ value & OR & $95 \% \mathrm{Cl}$ & $P$ value \\
\hline High aortic PP ( $\geq 50 \mathrm{~mm} \mathrm{Hg})$ & 2.05 & 1.69 to 2.50 & $<0.001$ & 1.42 & 1.13 to 1.78 & 0.003 \\
\hline Age $\geq 60$ years old & 2.50 & 2.01 to 3.10 & $<0.001$ & 1.79 & 1.40 to 2.30 & $<0.001$ \\
\hline Female sex & 1.57 & 1.27 to 1.93 & $<0.001$ & 1.29 & 1.03 to 1.61 & 0.027 \\
\hline Body mass index $\left(\geq 30 \mathrm{~kg} / \mathrm{m}^{2}\right)$ & 0.83 & 0.69 to 1.02 & 0.072 & 0.82 & 0.67 to 1.31 & 0.273 \\
\hline Hypertension & 1.35 & 1.11 to 1.63 & 0.002 & 1.07 & 0.86 to 1.33 & 0.547 \\
\hline Diabetes mellitus & 1.33 & 0.99 to 1.79 & 0.062 & 1.27 & 0.92 to 1.74 & 0.147 \\
\hline Dyslipidaemia & 1.25 & 1.03 to 1.15 & 0.025 & 1.06 & 0.86 to 1.31 & 0.573 \\
\hline Renal dysfunction (eGFR $<60 \mathrm{~mL} / \mathrm{min} / 1.73 \mathrm{~m}^{2}$ ) & 2.12 & 1.62 to 2.76 & $<0.001$ & 1.41 & 1.05 to 1.88 & 0.021 \\
\hline Current smoking & 0.62 & 0.45 to 0.85 & 0.003 & 0.77 & 1.40 to 2.30 & $<0.001$ \\
\hline
\end{tabular}

eGFR, estimated glomerular filtration rate; PP, pulse pressure.

\section{DISCUSSION}

This study demonstrates the association between directly assessed invasive aortic PP and coronary microvascular function and its prognostic implications. The main findings of this study were as follows: (1) High aortic PP was an independent determinant of CMEID, whereas CMED was not. The risk of CMEID increased non-linearly as aortic PP increased. Compared with the predictive models using the ROC curve analysis, IDI and NRI, the combination model that added aortic PP to risk factors was more predictive than the single predictive model of risk factors only for CMEID; (2) High aortic PP was an independent predictor of all-cause death; however, CMEID increased the risk of all-cause death only in patients with low aortic PP.

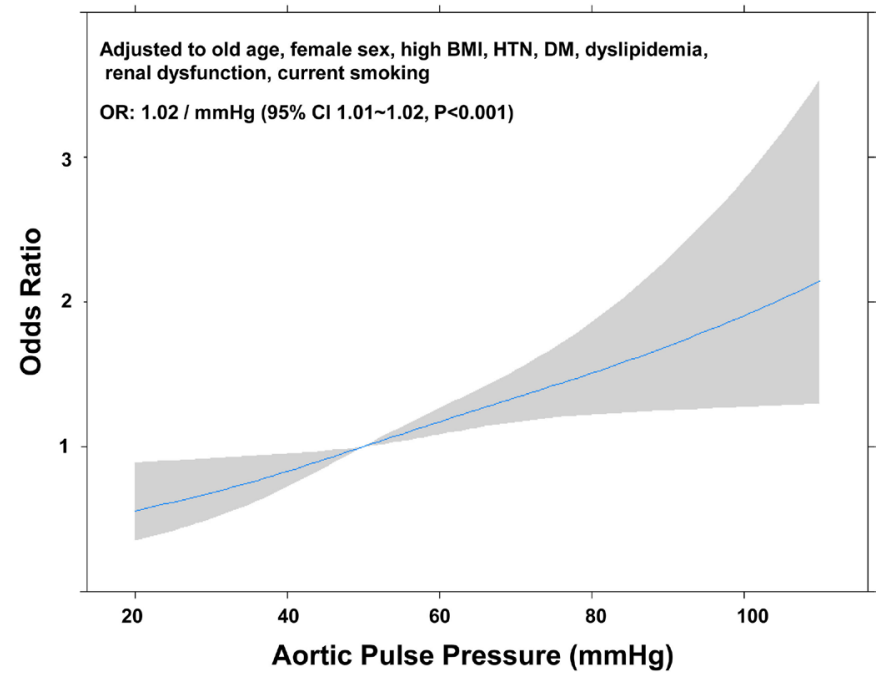

Figure 1 The estimated continuous OR of pulse pressures adjusted risk (OR; 95\% Cl) of pulse pressure for coronary microvascular endothelial independent dysfunction. Renal dysfunction, glomerular filtration rate $<60 \mathrm{~mL} / \mathrm{min} / 1.73$ $\mathrm{m}^{2}$ ). BMI, body mass index; DM, diabetes mellitus; HTN, hypertension.

\section{Arterial stiffness and coronary microvascular endothelial independent function}

CMD is mainly determined by a lack of response to endothelium-dependent and/or endothelium-independent vasodilation processes. ${ }^{17}$ Endothelium-independent dysfunction mainly occurs due to impaired myocyte tone and vascular smooth muscle cells (VSMCs). ${ }^{18}$ Two types of mechanical forces affect vessels: (1) shear stress and (2) cyclic strain of the vascular wall, which, according to Laplace's law, are mainly determined by cyclic changes in BP. ${ }^{19}$ Shear stress predominantly affects endothelial function; however, pulsatile cyclic changes in BP influence all structures of the arterial wall. The changes in intramural tension in the arterial wall are well recognised as important factors in progression

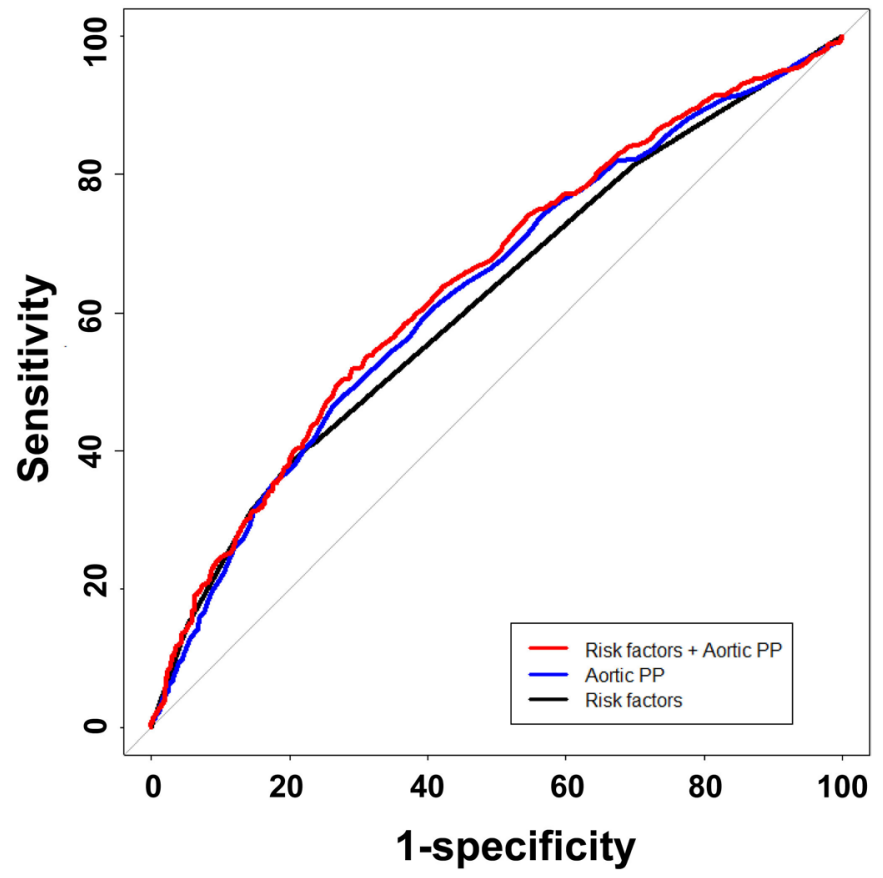

Figure 2 Receiver operating curve comparison comparison of receiver operating characteristic curves of risk factors and/ or intra-aortic pulse pressure (PP). 


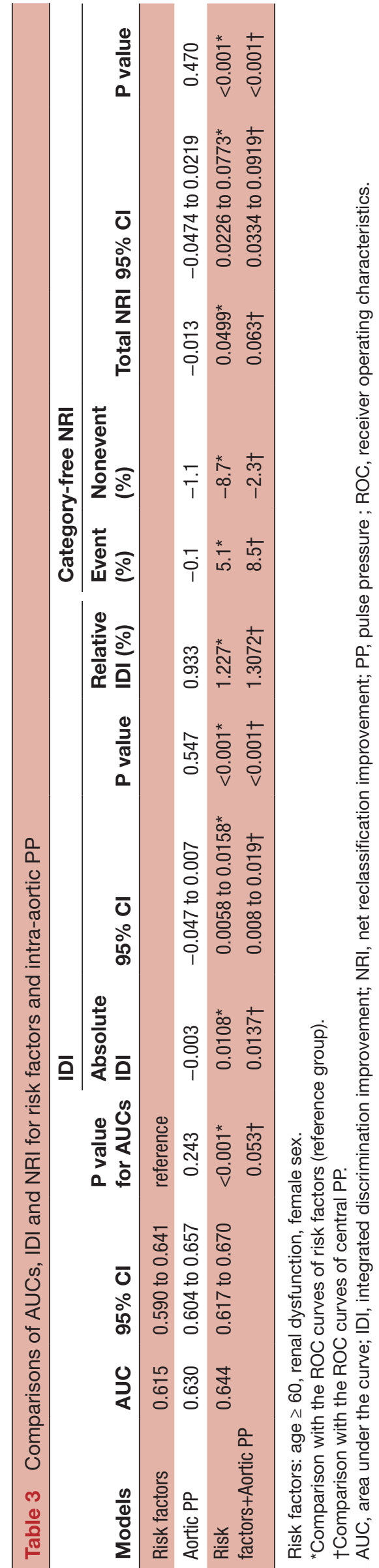

\begin{tabular}{|c|c|c|c|}
\hline Variables & HR & $95 \% \mathrm{Cl}$ & $P$ value \\
\hline CMEID & 1.25 & 0.88 to 1.78 & 0.211 \\
\hline $\begin{array}{l}\text { High aortic PP ( } \geq 50 \mathrm{~mm} \\
\mathrm{Hg} \text { ) }\end{array}$ & 2.08 & 1.34 to 3.32 & 0.002 \\
\hline Female sex & 0.46 & 0.32 to 0.66 & $<0.001$ \\
\hline Age $\geq 60$ & 2.79 & 1.85 to 4.19 & $<0.001$ \\
\hline $\begin{array}{l}\text { Body mass index }(\geq 30 \\
\left.\mathrm{kg} / \mathrm{m}^{2}\right)\end{array}$ & 1.02 & 0.71 to 1.47 & 0.913 \\
\hline Hypertension & 0.77 & 0.53 to 1.12 & 0.176 \\
\hline Diabetes mellitus & 2.10 & 1.31 to 3.37 & 0.002 \\
\hline Dyslipidaemia & 0.70 & 0.49 to 1.01 & 0.051 \\
\hline $\begin{array}{l}\text { Renal dysfunction } \\
(\mathrm{e} G F R<60 \mathrm{~mL} / \mathrm{min} / 1.73 \\
\left.\mathrm{m}^{2}\right)\end{array}$ & 1.87 & 1.25 to 2.79 & 0.002 \\
\hline Current smoking & 2.46 & 1.58 to 3.83 & $<0.001$ \\
\hline
\end{tabular}

CMEID coronary microvascular endothelial independent dysfunction; eGFR, estimated glomerular filtration rate; PP, pulse pressure.

of atherosclerosis. ${ }^{20}{ }^{21}$ The central artery is located near the target organs, and is more influential to them.

The large artery is composed of VSMCs and involves prominent elastin and collagen. It is highly sensitive to changes in age and BP. In large arteries, dysfunction of VSMCs are a key determinant of wall stiffness, whereas small vessels are highly sensitive to vasoreactive substances, particularly those of endothelial origin. ${ }^{22}$ The current study measured aortic PP in the ascending thoracic aorta as arterial stiffness, which is more dependent on the function of VSMCs. Unfortunately, the toolkit for assessing the stiffness of smaller muscular vessels is much less developed. CMEID also correlated with VSMC function, hence it is one cause for aortic PP being an independent determinant in the current study.

Arterial stiffness can lead to reduced coronary perfusion. Increasing arterial stiffness increases the propagating pulse wave velocity and backward wave returning to the heart, which can cause a wide PP. ${ }^{3}$ Therefore, the wave reflection is increased in arterial stiffness, and the reflection time is faster and reduces diastolic coronary perfusion pressure. ${ }^{2324}$ Kang et al reported that invasively measured aortic $\mathrm{PP}$ was strongly positively correlated with arterial stiffness as measured by pulse wave velocity in patients with $\mathrm{CAD} .{ }^{25}$

The basal myocardial flow can be enhanced in an aortic stiffness experimental model, primarily due to the augmentation of coronary flow during systole. ${ }^{26}$ This study also showed that the high aortic PP group had a high base APV (26.6 vs $23.5 \mathrm{~cm} / \mathrm{s}, \mathrm{p}<0.001)$ compared with the low PP group.

Increased arterial stiffness leads to increased cardiac work and increased myocardial oxygen demand. ${ }^{6}$ Aortic stiffening increases afterload and left ventricle work load, resulting in left ventricular hypertrophy (LVH). Chung 
A
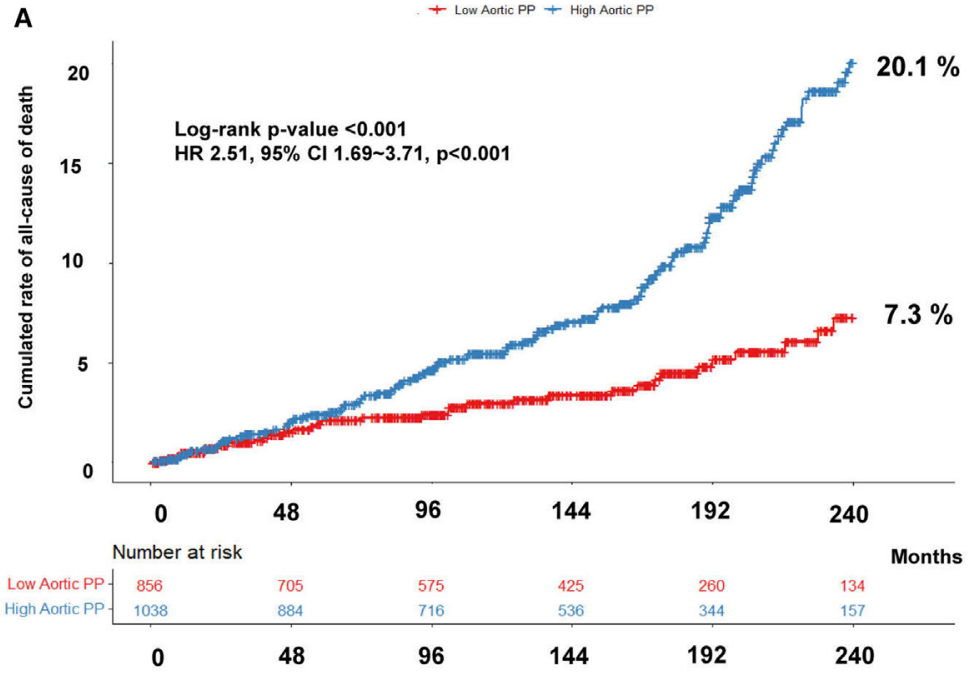

B - CMEID $(-)+$ CMEIO $(+)$

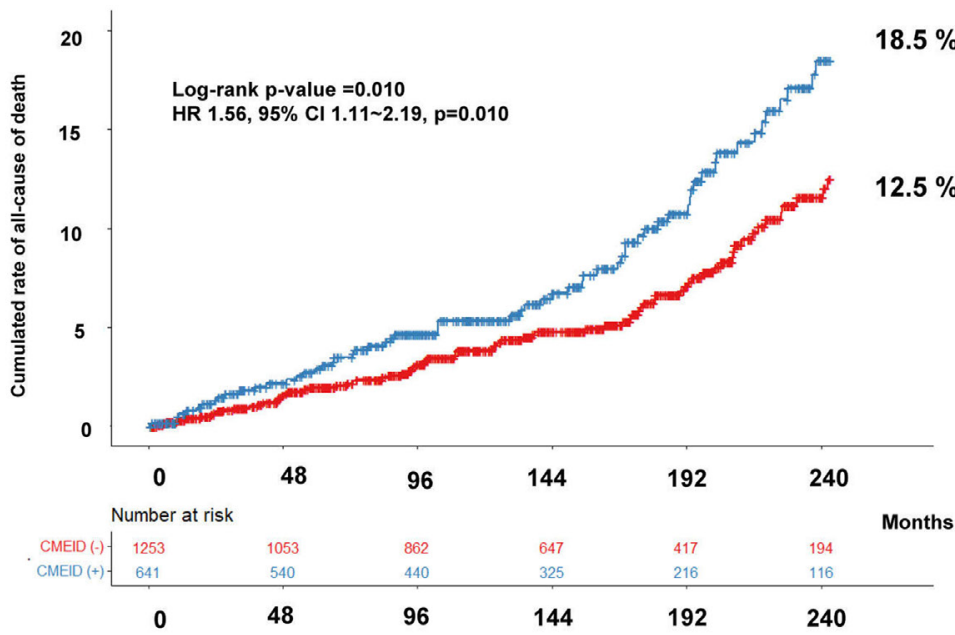

C + Low PP \& CMEID $(-)$ + Low PP \& CMEID $(+)+\operatorname{High}$ PP \& CMEID $(-)+\operatorname{High}$ PP \& CMEID $(+)$

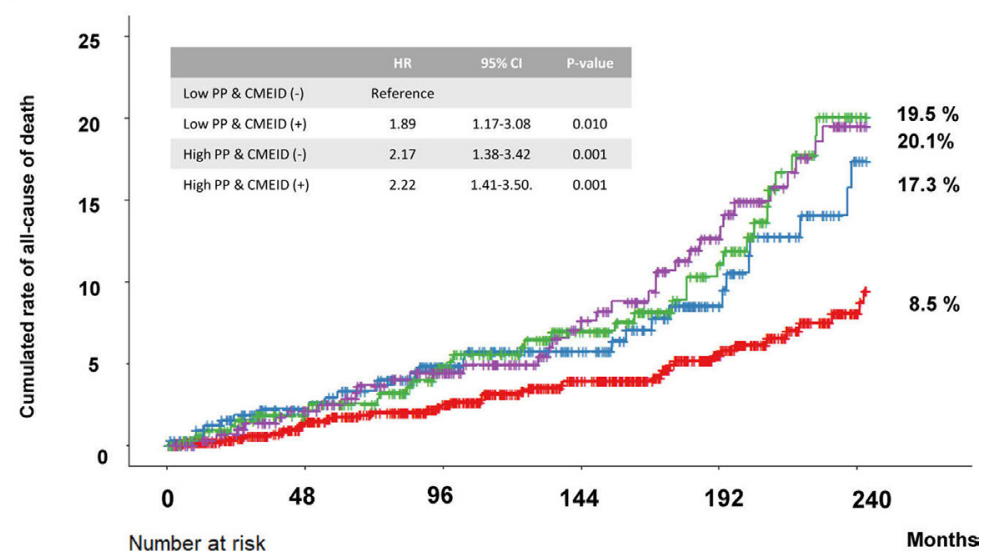

\begin{tabular}{lcccccc} 
Low PP \& CMEID (-) & 910 & 760 & 620 & 473 & 302 & 137 \\
LOW PP \& CMED (+) & 335 & 276 & 220 & 158 & 95 & 47 \\
High PP \& CMED (-) & 343 & 293 & 242 & 174 & 115 & 57 \\
High PP \& CMEID (+) & 306 & 264 & 220 & 167 & 121 & 69 \\
\hline & $\mathbf{0}$ & $\mathbf{4 8}$ & $\mathbf{9 6}$ & $\mathbf{1 4 4}$ & $\mathbf{1 9 2}$ & $\mathbf{2 4 0}$
\end{tabular}

Figure 3 Kaplan-Meier curves; (A) groups as per intra-aortic PP for all-cause of death, (B) groups as per CMEID for allcause death, (C) groups as per intra-aortic PP and CMEID for all-cause of death. CMEID, coronary microvascular endothelial independent dysfunction; PP, pulse pressure. 
et al reported that pulse wave velocity was significantly associated with $\mathrm{LVH}$, and large artery stiffening seemed to significantly contribute to LVH pathogenesis. ${ }^{27} \mathrm{LVH}$ may increase microvascular resistance and impair the CFR. CFR abnormalities have been observed in patients with LVH. ${ }^{28}$ The current study did not collect the data as to whether participants had LVH; however, it revealed that HMR was increased in the higher aortic PP group. Muroya et al also reported that aortic stiffness (assessed using the cardio-ankle vascular index) was an independent determinant of the invasive HMR index $(\beta=0.25$, $\mathrm{p}=0.007) .{ }^{29}$ The high aortic PP group had a high ASCVD risk score and was more likely to have comorbidities such as DM, HTN and dyslipidaemia. Although none of the patients had obstructive coronary disease, many patients may have minimal artery disease, and the high aortic PP group may more likely demonstrate minimal stenosis compared with patients with low aortic PP. HMR is defined as mean distal coronary artery pressure divided by average distal APV at maximal hyperaemia, and BMR is defined as MAP divided by APV at baseline ${ }^{15}{ }^{16}$ If patients have minimal stenosis, HMR can be increased by reduced distal flow and velocity; therefore, it is possible that HMR may be overestimated in patients with high PP. HMR has been debated in patients with coronary artery stenosis because it is overestimated in the presence of coronary artery stenosis compared with real microvascular resistance because collateral flow is not considered. ${ }^{30}$ CFR is obtained based on the hyperaemic flow velocity and baseline resting flow velocity ratio using the Doppler wire technique. ${ }^{1031}$ Baseline APV was higher in higher PP group, but flow velocity was not sufficient increased in hyperaemic state compared with lower aortic PP patients. Therefore, HMR and CFR can be overestimated and/or underestimated in high aortic PP.

Several studies have reported that increased aortic stiffness is independently associated with CMD in patients with non-obstructive CAD. Lembo et al reported that patients with angiographically non-obstructive CAD had a higher resting coronary flow velocity and lower CFR. This study examined transthoracic-derived CFR in a study population of 398 patients. However, the resting coronary flow velocity, and not the CFR, was significantly associated with PP and only on multiple linear regression analysis, at that. ${ }^{9}$ Other echo-based studies have also demonstrated that arterial stiffness is inversely related to CFR or hyperaemic coronary flow velocity in patients with nonobstructive CAD. ${ }^{7-9}$ Previous studies used transthoracic echocardiography to measure coronary flow and velocity, and recruited a relatively small number of participants. These studies did not measure invasive aortic BP.

This current study, on the other hand, measured coronary vasoreactivity using invasive Doppler wire, and the number of participants was $>1800$ patients. Invasive aortic $\mathrm{PP}$ was determined for all patients. Central PP more accurately reflects vascular load on the left ventricle and coronary vasculature, and, furthermore, central PP is more correlated with target organ damage and cardiovascular outcomes than is brachial PP. ${ }^{11}$ This study also suggested that old age, female sex, renal dysfunction and current smoking were independent determinants of CMEID.

\section{Arterial stiffness and coronary endothelial function}

Coronary endothelial dysfunction is the earliest stage of coronary atherosclerosis and is also a vascular dysfunction along with arterial stiffness, associated with the development of atherosclerotic disease, resulting in cardiovascular events. ${ }^{32}$

Oxidative stress and chronic inflammation lead to endothelial dysfunction, leading to functional stiffness due to reduced endothelial nitric oxide production. ${ }^{33}$ Peripheral endothelial function is usually assessed using flow-mediated dilatation or peripheral arterial tonometry. Beigel et al reported that arterial stiffness (PP) was inversely associated with brachial FMD. ${ }^{34}$ However, there have been no studies on the relationship between peripheral arterial stiffness and coronary endothelial function.

The current study showed that high aortic PP did not correlate with CMED. Accordingly, aortic arterial stiffness may not be directly associated with coronary endothelial function. The current study revealed that old age, BMI and current smoking were independent determinants of CMED.

\section{PP and CMEID for all-cause of death}

Functional coronary microvascular dysfunction results in ischaemic symptoms, adverse cardiovascular events and increased cardiovascular mortality. ${ }^{2}$ Several studies have reported that high PP (as arterial stiffness) increases adverse clinical outcomes and death. Roman et al suggested that a high central PP is independently associated with adverse cardiovascular outcomes. ${ }^{45}$ Consistent with other studies, the current study also showed that high aortic PP increased the risk of all-cause death in non-obstructive CAD patients. Among patients with low aortic PP, CMEID increased the risk of all-cause death in the multivariate analysis in patients with non-obstructive CAD.

\section{Limitations}

This was a single-centre, retrospective cohort study. Enrolled patients were referred for coronary angiography to a tertiary referral centre by an independent cardiologist and thus constituted a unique population, which may limit its generalisability. Second, we did not use intravascular imaging to evaluate the grade of atherosclerosis; therefore, we could not compare coronary atherosclerosis and CMD. Third, cardiovascular adverse outcomes are important during follow-up in non-obstructive CAD patients. However, data collection was performed retrospectively, and cardiovascular outcome data could not be attained, it was obtained all-cause mortality information. Fourth, HMR could be overestimated in patients with stenotic coronary disease even if there is minimal stenosis; however, we did not collect data on stenosis among our cohort. We also did not determine whether LVH was 
present, which is one of the causes of the increase in HMR. Finally, we could not obtain data on follow-up BP status and maintenance medications. However, this study had the highest number of patients undergoing an invasive coronary vasoreactivity test performed by expert physicians, and aortic PP was measured invasively in this case at the ascending thoracic aorta. Furthermore, mortality data from all patients were collected in this study.

\section{CONCLUSIONS}

Our study attempted to identify the association between aortic PP, arterial stiffness and CMEID in patients with non-obstructive CAD. Whether choosing medical treatment based on measurements of these risk factors can improve clinical outcomes in non-obstructive CAD patients requires further study.

Acknowledgements The authors appreciate the help of Faten Sebaali (FS) in data collection.

Contributors PHW: conceptualisation, methodology, formal analysis, investigation, writing — original draft and visualisation, writing - review and editing. MC: conceptualisation, methodology and writing—review and editing. TT: investigation and writing — review and editing. Ilke 0zcan: writing—review and editing. LL: conceptualisation, methodology and writing — review and editing. AL: conceptualisation, methodology, resources and writing — review and editing, guarantor. PHW, AA take full responsibility for the integrity of the data and the accuracy of the data analysis.

Funding This work was funded by the Mayo Foundation.

Competing interests $\mathrm{AL}$ is a member of the advisory board of Itamar Medical, a company that produces EndoPAT, a device for noninvasive endothelial function detection. This device was not used in this study. LL is his spouse.

Patient consent for publication Consent obtained directly from patient(s).

Ethics approval This study complied with the Declaration of Helsinki guidelines and was approved by the Mayo Clinic Institutional Review Board (05-004191). Participants gave informed consent to participate in the study before taking part.

Provenance and peer review Not commissioned; externally peer reviewed.

Data availability statement № additional data are available.

Open access This is an open access article distributed in accordance with the Creative Commons Attribution Non Commercial (CC BY-NC 4.0) license, which permits others to distribute, remix, adapt, build upon this work non-commercially, and license their derivative works on different terms, provided the original work is properly cited, appropriate credit is given, any changes made indicated, and the use is non-commercial. See: http://creativecommons.org/licenses/by-nc/4.0/.

\section{ORCID iDs}

Lilach Lerman http://orcid.org/0000-0002-3271-3887

Amir Lerman http://orcid.org/0000-0002-9446-5313

\section{REFERENCES}

1 Radico F, Cicchitti V, Zimarino M, et al. Angina pectoris and myocardial ischemia in the absence of obstructive coronary artery disease: practical considerations for diagnostic tests. JACC Cardiovasc Interv 2014;7:453-63.

2 Fearon WF, Low AF, Yong AS, et al. Prognostic value of the index of microcirculatory resistance measured after primary percutaneous coronary intervention. Circulation 2013;127:2436-41.

3 O'Rourke M, Frohlich ED. Pulse pressure: is this a clinically useful risk factor? Hypertension 1999;34:372-4.

4 Roman MJ, Devereux RB, Kizer JR, et al. Central pressure more strongly relates to vascular disease and outcome than does brachial pressure: the strong heart study. Hypertension 2007;50:197-203.

5 Roman MJ, Ganau A, Saba PS, et al. Impact of arterial stiffening on left ventricular structure. Hypertension 2000;36:489-94.

6 WW N, OR MF. McDonald's Blood Flow in Arteries. 4th ed. London, UK: Arnold, 1998.
7 Saito M, Okayama H, Nishimura K, et al. Possible link between large artery stiffness and coronary flow velocity reserve. Heart 2008;94:e20.

8 Mahfouz RA, Elawady W, Abdu M, et al. Associations of fractional pulse pressure to aortic stiffness and their impact on diastolic function and coronary flow reserve in asymptomatic diabetic patients with normal coronary angiography. Cardiol $J$ 2013;20:605-11.

9 Lembo M, Sicari R, Esposito R, et al. Association between elevated pulse pressure and high resting coronary blood flow velocity in patients with angiographically normal epicardial coronary arteries. J Am Heart Assoc 2017;6. doi:10.1161/JAHA.117.005710. [Epub ahead of print: 29 Jun 2017].

10 Widmer RJ, Samuels B, Samady H, et al. The functional assessment of patients with non-obstructive coronary artery disease: expert review from an international microcirculation working group. Eurolntervention 2019;14:1694-702.

11 Roman MJ, Devereux RB, Kizer JR, et al. High central pulse pressure is independently associated with adverse cardiovascular outcome the strong heart study. J Am Coll Cardiol 2009;54:1730-4.

12 Toya T, Corban MT, Park JY, et al. Prognostic impact and clinical outcomes of coronary flow reserve and hyperaemic microvascular resistance. Eurolntervention 2021;17:569-75.

13 Ong P, Camici PG, Beltrame JF, et al. International standardization of diagnostic criteria for microvascular angina. Int J Cardiol 2018;250:16-20.

14 Díez-Delhoyo F, Gutiérrez-Ibañes E, Fernández-Avilés F. Functional disorders in non-culprit coronary arteries and their implications in patients with acute myocardial infarction. Trends Cardiovasc Med 2020;30:346-52.

15 Chamuleau SAJ, Tio RA, de Cock CC, et al. Prognostic value of coronary blood flow velocity and myocardial perfusion in intermediate coronary narrowings and multivessel disease. J Am Coll Cardiol 2002;39:852-8.

16 Dhawan SS, Corban MT, Nanjundappa RA, et al. Coronary microvascular dysfunction is associated with higher frequency of thin-cap fibroatheroma. Atherosclerosis 2012;223:384-8.

17 Lerman A, Zeiher AM. Endothelial function: cardiac events. Circulation 2005;111:363-8.

18 Gutterman DD, Chabowski DS, Kadlec AO, et al. The human microcirculation: regulation of flow and beyond. Circ Res 2016;118:157-72.

19 Fuster V, Lewis A, Lecture CM. Lewis A. Conner memorial lecture. mechanisms leading to myocardial infarction: insights from studies of vascular biology. Circulation 1994;90:2126-46.

20 Shaw A, Xu Q. Biomechanical stress-induced signaling in smooth muscle cells: an update. Curr Vasc Pharmacol 2003;1:41-58.

21 Yamamoto K, Ikeda U, Shimada K. Role of mechanical stress in monocytes/macrophages: implications for atherosclerosis. Curr Vasc Pharmacol 2003;1:315-9.

22 Safar ME, Levy BI, Struijker-Boudier H. Current perspectives on arterial stiffness and pulse pressure in hypertension and cardiovascular diseases. Circulation 2003;107:2864-9.

23 Baumbach GL. Effects of increased pulse pressure on cerebral arterioles. Hypertension 1996;27:159-67.

24 James MA, Watt PA, Potter JF, et al. Pulse pressure and resistance artery structure in the elderly. Hypertension 1995;26:301-6.

25 Kang J, Kim H-L, Lim W-H, et al. Relationship between brachialankle pulse wave velocity and invasively measured aortic pulse pressure. J Clin Hypertens 2018;20:462-8.

26 Kass DA, Saeki A, Tunin RS, et al. Adverse influence of systemic vascular stiffening on cardiac dysfunction and adaptation to acute coronary occlusion. Circulation 1996;93:1533-41.

27 Chung C-M, Lin Y-S, Chu C-M, et al. Arterial stiffness is the independent factor of left ventricular hypertrophy determined by electrocardiogram. Am J Med Sci 2012;344:190-3.

28 Gimelli A, Schneider-Eicke J, Neglia D, et al. Homogeneously reduced versus regionally impaired myocardial blood flow in hypertensive patients: two different patterns of myocardial perfusion associated with degree of hypertrophy. J Am Coll Cardiol 1998;31:366-73.

29 Muroya T, Kawano H, Koga S, et al. Aortic stiffness is associated with coronary microvascular dysfunction in patients with nonobstructive coronary artery disease. Intern Med 2020;59:2981-7.

30 Nolte F, van de Hoef TP, Meuwissen M, et al. Increased hyperaemic coronary microvascular resistance adds to the presence of myocardial ischaemia. Eurolntervention 2014;9:1423-31.

31 Sara JD, Widmer RJ, Matsuzawa Y, et al. Prevalence of coronary microvascular dysfunction among patients with chest pain and nonobstructive coronary artery disease. JACC Cardiovasc Interv 2015;8:1445-53. 
32 Higashi Y, Noma K, Yoshizumi M, et al. Endothelial function and oxidative stress in cardiovascular diseases. Circ J 2009;73:411-8.

33 Kataoka H, Murakami R, Numaguchi Y, et al. Angiotensin II type 1 receptor blockers prevent tumor necrosis factor-alpha-mediated endothelial nitric oxide synthase reduction and superoxide production in human umbilical vein endothelial cells. Eur $J$ Pharmacol 2010;636:36-41.
34 Beigel R, Dvir D, Arbel Y, et al. Pulse pressure is a predictor of vascular endothelial function in middle-aged subjects with no apparent heart disease. Vasc Med 2010;15:299-305.

35 Park HW, Kang MG, Kim K, et al. Association between pulse pressure at discharge and clinical outcomes in patients with acute myocardial infarction: from the KAMIR-Korean-NIH registry. J Clin Hypertens 2019;21:774-85. 